\title{
Motion perception over long interstimulus intervals
}

\author{
PETER J. BEX and CURTIS L. BAKER, JR. \\ McGill University, Montreal, Quebec, Canada
}

\begin{abstract}
Recent studies using moving arrays of textured micropatterns have suggested that motion perception can be supported by two mechanisms, one quasilinear and sensitive to the motion of luminancedefined local texture, the other nonlinear and coding motion of contrast-defined envelopes of texture (Baker \& Hess, 1998; Boulton \& Baker, 1993b). Here we used similar patterns to study motion perception under conditions previously shown to isolate the nonlinear mechanism (low micropattern densities and positive interstimulus intervals [ISIs]). We measured direction discrimination for two-flash apparent motion over a much larger range of ISIs, and susceptibility to masking by incoherently moving "distractor" micropatterns. The results suggest that two nonlinear mechanisms can support motion perception under these conditions. One operates only for relatively short ISIs (less than c. $100 \mathrm{msec}$ ), is sensitive to small spatial displacements, and is relatively insensitive to distractor masking. The other operates over much longer ISIs, is insensitive to small spatial displacements, and is highly disrupted by distractor masking. These results are in line with previous studies suggesting that three mechanisms support motion perception.
\end{abstract}

Although the perception of movement might be thought to be mediated by an appreciation of the displacement of identifiable objects to novel positions at successive times, motion perception is now largely understood to be mediated by low-level neural mechanisms (for review, see Nakayama, 1985). This belief is supported by findings as diverse as the motion aftereffect, neurophysiological direction selectivity of single neurons, and the specific impairment of motion perception following brain damage (Zihl, Von Cramon, \& Mai, 1983). Recent psychophysical evidence favors the existence of two motion detection systems, one based on linear spatiotemporal summation that responds to "first-order" (or "Fourier") motion of luminance-defined contours, and the other ("secondorder," or "non-Fourier") based on a nonlinear extraction of overall envelopes of other stimulus attributes, such as local contrast (Chubb \& Sperling, 1988). Low-level linear models of motion detection (e.g., Adelson \& Bergen, 1985; van Santen \& Sperling, 1985; Watson \& Ahumada, 1985) employ local motion analyzers selective for a particular spatial and temporal frequency of motion. Such "motion energy" detectors respond to the displacements of a variety of images but are incapable of signaling motion of certain second-order or non-Fourier motion stimuli that human observers reliably see as moving (Chubb \& Sperling, 1988).

It is possible that the motion of non-Fourier stimuli could be detected by a high-level "feature-tracking" mechanism that calculates the positional changes over time of

\footnotetext{
We are grateful to Isabelle Mareschal for observing. We also thank Michael Moskovich and Ken Charles for contributions to computer programming. This research was supported by Canadian NSERC Grant OGPOOO1978 to C.L.B. Correspondence should be addressed to P. J. Bex, Department of Psychology, University of Essex, Wivenhoe Park, Colchester C04 3SQ, England (e-mail: bex@psy.gla.ac.uk).
}

salient features in a stimulus (see, e.g., Palmer, 1986; Ullman, 1979). However, physiological recordings from primates (Albright, 1992) and cats (Zhou \& Baker, 1993) and psychophysical evidence that motion aftereffects may be elicited by adaptation to non-Fourier motion stimuli (Ledgeway \& Smith, 1995; Nishida \& Sato, 1995) support the notion that second-order motion is analyzed by low-level motion detectors. Nishida (1993) has shown that reversed-phi motion occurs for non-Fourier stimuli, suggesting low-level detection, while Smith (1994) has argued that non-Fourier motion is detected by a low-level mechanism in some circumstances and by feature tracking in others. Several psychophysical studies (e.g., Lu \& Sperling, 1995a, 1995b; Mather \& West, 1993; Nishida, Ledgeway, \& Edwards, 1997) suggest that the nonlinear low-level mechanism is not the same as that which detects first-order or Fourier motion. Thus, there appear to be three motion mechanisms in total, two low level and one high level. Several models have been proposed in which motion can be detected by conventional motion energy detectors after a nonlinear transformation of the image (e.g., Chubb \& Sperling, 1988; Werkhoven, Sperling, \& Chubb, 1993; Wilson, Ferrara, \& Yo, 1992). The nonlinear stage typically follows initial band-pass spatial frequency filtering and utilizes an intensive nonlinearity such as full-wave or half-wave rectification.

Support for distinct quasilinear and nonlinear motion mechanisms has come from studies using random kinematograms in which a field of pseudorandomly positioned micropatterns (Figure 1A) are abruptly shifted between two or more presentations of the stimulus (Baker \& Hess, 1998; Boulton \& Baker, 1993a, 1993b, 1994). The micropatterns are Gabor functions (small patches of sine wave "carrier" grating, each enclosed in a smooth Gaussian envelope), whose construction allows independent manipulation of spatial frequency and density. The spatio- 

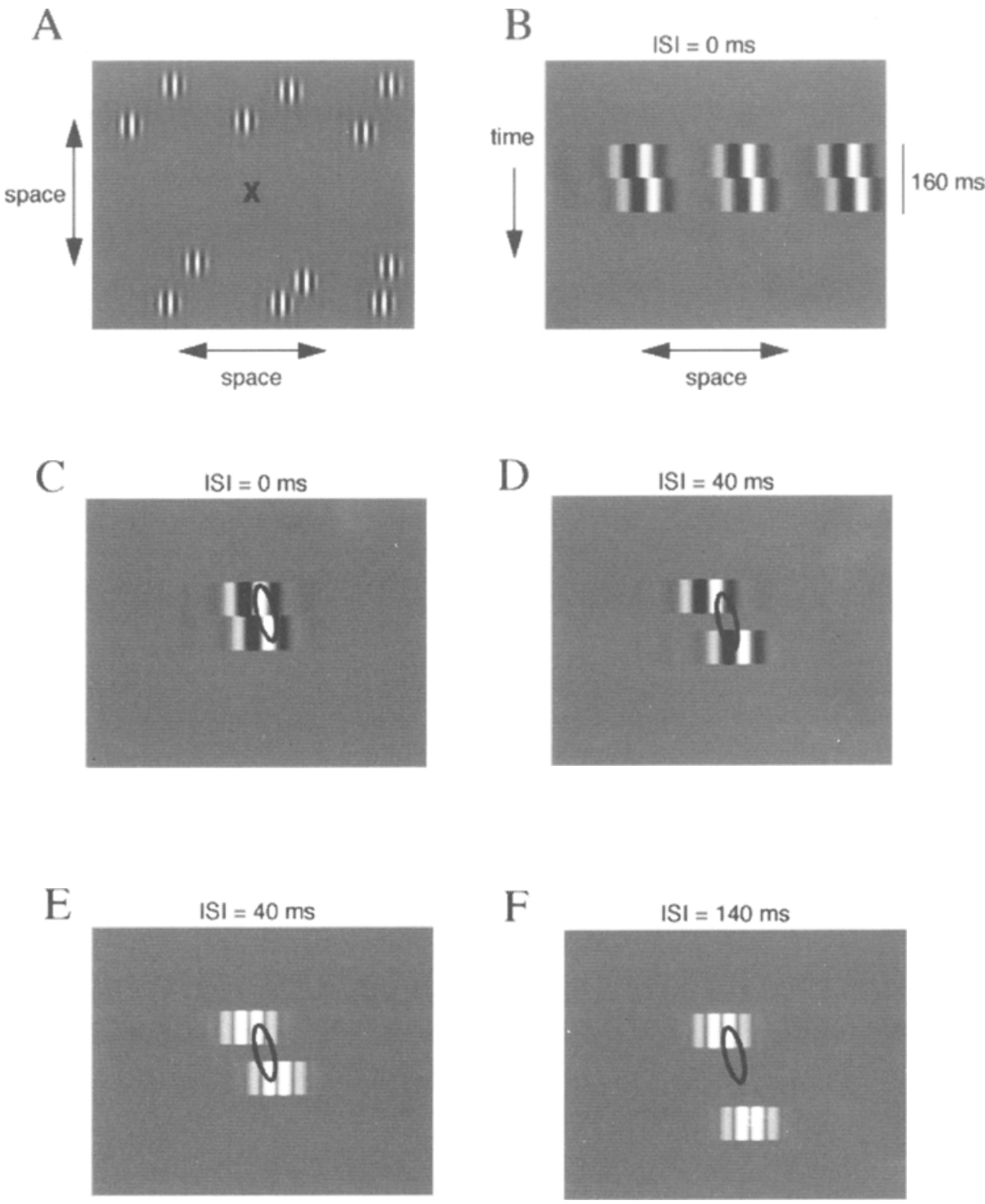

Figure 1. Spatiotemporal depictions of stimulus and actions of putative motion mechanisms. A: Spatial layout of stimulus, consisting of two rows of pseudorandomly placed Gabor micropatterns, placed in strips above and below a central fixation cross. B: Example of a space-time diagram for a spatial profile through a single row of Gabor micropatterns, graphed with time running downward, showing an apparent motion presentation with flashes each $80 \mathrm{msec}$, and interstimulus interval $($ ISI $)=0$. C: Space-time diagram for a single micropattern (ISI $=0$ ), with a superimposed ellipse to schematically illustrate the linear summation area of a spatiotemporally oriented filter; the net summation will be relatively large, giving a good motion signal. D: As for C, but for larger ISI (40 msec) and larger displacement, in which net summation will be very small. E: As for $D$, but with full-wave rectification, now producing large summation. F: As for E, but at larger ISI, now producing poor summation.

temporal nature of the stimulus can be illustrated in a "space-time diagram" in which a spatial luminance profile is graphed as a function of time (ordinate); the gray level indicates the luminance of the stimulus at each point in space and time. Figure 1B shows such a space-time diagram for one example in which three Gabor functions are presented for $80 \mathrm{msec}$ at each position, with ISI $=0$.
On the basis of psychophysical performance in direction discrimination using such stimuli, Boulton and Baker (1993b) interpreted their results in terms of two hypothetical mechanisms of motion detection: a quasilinear mechanism with a half-cycle displacement limit operating at high element densities and short stimulus onset asynchronies (SOAs), which preserves and is limited by 
the fine-grain texture of the micropattern carrier; and a nonlinear mechanism isolated at lower element densities and long SOAs, which responds to motion of the envelopes while discarding carrier information.

For a theoretical overview of motion mechanisms in the context of this paper, it is useful to consider a spacetime diagram of such an apparent motion stimulus, for clarity illustrating only a single Gabor micropattern. Figure $1 \mathrm{C}$ shows such a plot for an ISI $=0$ (SOA $=$ $80 \mathrm{msec}$ ), in which the micropattern is displaced on the second presentation by one quarter of a cycle of the carrier sine wave. Superimposed on the stimulus is a tilted ellipse, schematically illustrating part of a "space-time oriented filter" (Adelson \& Bergen, 1985), which linearly summates local luminances with a time lag whose extent varies with spatial position; in this example such a filter would give a strong directional response (compared with an identical filter, tilted in the opposite direction of motion), because like-signed parts of the stimulus add synergistically within its summation region. However, at larger temporal and spatial displacements (Figure 1D, ISI $=40 \mathrm{msec}$ ), the stimulus no longer aligns in so favorable a manner with the filter, and linear summation of positive and negative regions produces little or no response. Thus such a quasilinear motion model predicts best performance at smaller spatial and temporal separations, with a maximum displacement, $d_{\max }$, approximately equal to one-half cycle of the carrier sine wave grating - the "half-cycle limit" of motion energy detectors (Bischof \& Di Lollo, 1991). A typical nonlinear model of motion detection utilizes an intensive nonlinearity prior to the motion detection operation; for example, Figure $1 \mathrm{E}$ shows a full-wave rectification (absolute value operation), which extracts the overall envelope of the Gabor micropattern while discarding information about the fine texture of the carrier waveform. This model allows stimulus regions, which are now like-signed, to summate in a subsequent linear space-time oriented filter. Such a model predicts a $d_{\max }$ that is much greater than a spatial scale of the sine wave carrier, instead dependent on the density of micropattern envelopes. Although such a nonlinear motion mechanism evidently uses spacetime filters tuned to larger temporal separations (Boulton \& Baker, 1993a), nevertheless at still larger time separations (Figure 1F, ISI $=140 \mathrm{msec}$ ) such a nonlinear motion energy mechanism would be expected to fail. Details of computer simulations of how such linear and nonlinear motion energy models respond to two-flash Gabor kinematograms can be found in Bex and Baker (1997).

In pilot studies, we noticed that it was possible to report correctly the direction of displacement of low-density, random Gabor kinematograms over exceptionally long ISIs (e.g., 1,000 msec or more). Unlike at the shorter ISIs previously studied, these stimuli do not always provide a genuine subjective sensation of "motion," but instead may appear at different identifiable positions at successive times. For example, in the limiting case it is possible to indicate the direction of displacement of a single patch that has been shifted from the left edge of the display to the right as long as the observer can remember that the patch was originally on the left, but this is not necessarily accompanied by the subjective appearance of movement. Here we report measurements of direction discrimination performance for low-density random Gabor kinematograms over a much greater range of ISIs than previously explored. To test the idea that performance at very long ISIs could be mediated by a high-level feature-tracking operation (Ullman, 1979) or a perceived change of position (Palmer, 1986), rather than by a low-level motion detection mechanism, we measured the impact of adding "distractor" micropatterns that are identical in spatial structure to the targets, but were not coherently displaced. Performance of a low-level, nonlinear, energy-based (second-order) mechanism should be only moderately degraded by distractor masking, as in Bex and Baker (1997), whereas a high-level recognition of positional change would be severely disrupted by the ambiguity of correspondence introduced by the distractors. Smith (1994) used the same logic in the context of periodic nonFourier patterns and found that a masking pattern disrupted performance for long but not short ISIs.

\section{GENERAL METHOD}

Stimuli were generated using a VSG $2 / 1$ graphics card (Cambridge Research Systems) in a host PC microcomputer (386$33 \mathrm{MHz}$ ), and were presented on a Nanao Flexscan 6500 monitor with P4 phosphor and a frame rate of $118 \mathrm{~Hz}$. The image was $512 \times$ 428 pixels, which subtended $16.0^{\circ} \times 13.4^{\circ}$ at the viewing distance of $118 \mathrm{~cm}$. The mean luminance of the display was $32 \mathrm{~cd} / \mathrm{m}^{2}$ and luminance was calibrated with a United Detector Technology, S370 photometer. The luminance $z$-nonlinearity of the display was linearized with pseudo-12-bit resolution (Pelli \& Zhang, 1991) by resistively adding the color video signals with an ISR Video Attenuator (Institute for Sensory Research, Syracuse University, Syracuse, NY) and using VideoToolbox (C1996) software (Pelli, 1997) adapted for use on a PC. Pseudo-12-bit resolution in this case allowed the presentation of $2^{8}$ gray levels from a possible range of $2^{12}$ levels.

\section{Stimuli}

The stimuli were composed of arrays of Gabor function micropatterns; each Gabor was a one-dimensional sine wave grating (carrier), multiplied by a two-dimensional Gaussian envelope:

$$
L_{(x, y)}=L_{0}\left\{1+C_{\text {exp }}\left[-x^{2} / 2 \sigma^{2}+y^{2} / 2 \sigma^{2}\right] * \sin (2 \pi x / \lambda)\right\}
$$

where $L_{0}=$ mean luminance; $C=$ contrast; $\sigma=$ width parameter of Gaussian envelope; $\lambda=$ spatial wavelength (reciprocal of carrier spatial frequency). The spatial frequency of the sine wave carrier was $2.0 \mathrm{c} / \mathrm{deg}\left(\lambda=0.5^{\circ}\right)$ and $\sigma=0.75 \lambda$. The carrier was always in sine phase to ensure that mean luminance of the Gabor was identical to the background. The contrast of the Gabors, defined as $\left(L_{\max }\right.$ $\left.-L_{\min }\right) /\left(L_{\max }+L_{\min }\right)$ was $25 \%$ in all conditions. The micropatterns were placed in two strips across the top and bottom of the stimulus field (Figures $1 \mathrm{~A}$ and $4 \mathrm{~A}$ ), approximately $2^{\circ}$ above and below the fixation cross, to confine the stimulus in eccentricity and to prevent the observers from paying attention to a fortuitous stimulus "fea- 
ture" close to the fixation point (e.g., a relatively isolated micropattern). Within each of these strips, the micropatterns were placed in such a way as to ensure a relatively uniform distribution, while avoiding periodicity or clustering; this was achieved by defining a notional grid of equally spaced locations along two rows in each strip. The location of each micropattern in each row was randomly shifted horizontally and vertically within $\pm 1.2^{\circ}$, and the whole row was then shifted within $\pm 8^{\circ}$ horizontally. The micropatttern locations were rerandomized every trial. Wrap-around was employed at the display boundaries.

We used stimulus parameters shown in previous work (Boulton \& Baker, 1993a, 1993b) to preclude quasilinear motion detection, while allowing motion based on the micropattern envelopes (i.e., a nonlinear mechanism): low target density, relatively long SOA, and displacement greater than the spatial scale of the micropattern carrier. In the present experiments, the exposure of each flash was $80 \mathrm{msec}$ in all cases and an ISI of variable duration was presented between flashes. During the ISI, as well as the intertrial intervals, the screen was a blank field at the mean luminance $\left(L_{0}\right)$ of the display. The element density was three targets per row (12 per flash) so that a small number of extra micropatterns could be added without producing too high a density for nonlinear behavior.

\section{Procedure}

Observers were seated at the required viewing distance and were instructed to fixate a small spot in the center of the screen. The screen was a blank field of mean luminance at all times except during stimulus presentations. Observers pressed response keys to initiate a two-flash apparent motion sequence and to indicate the direction of motion, left or right. Before the beginning of data collection, observers practiced the task with visual feedback; during data collection there was no feedback. Direction discrimination was measured for a range of displacements that were set according to a method of constant stimuli to cover the range from fewest to most errors. There were five levels, each presented 10 times in random order on each run, with four runs for each condition. All observers had normal or corrected-to-normal acuity and viewed the display binocularly.

\section{EXPERIMENT 1 \\ Upper and Lower Displacement Limits as a Function of ISI}

We first measured two simple indicators of motion performance, the maximum displacement $\left(d_{\max }\right)$ and the minimum displacement $\left(d_{\min }\right)$ for accurate direction discrimination, as a function of ISI. For the measurements of $d_{\max }$, a vertical orientation of the carrier grating in the Gabor micropatterns was used. In pilot studies it was found that the orientation did not affect the results, which is consistent with the proposal that a nonlinear motion mechanism responds to the micropattern envelope rather than the oriented sinusoidal carrier. In order to ensure that the estimate of $d_{\min }$ was based on the displacement of the envelope and not the carrier, the Gabor micropatterns were aligned orthogonal to the axis of motion (i.e., they had a horizontally oriented carrier). A psychometric function (Weibull, 1951) was fitted to the data for each of the four blocks of trials for each ISI, and $d_{\max }$ and $d_{\min }$ were inferred as the displacements, giving $20 \%$ errors. Means and standard errors of these estimates were then calculated across the trial blocks.

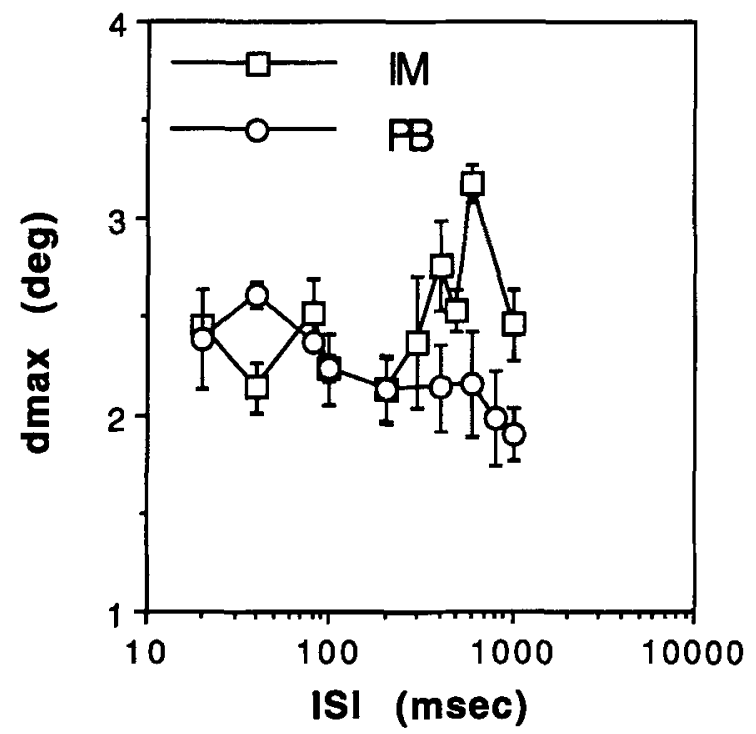

Figure 2. $d_{\max }$ as a function of interstimulus interval (ISI) for 2 observers. $d_{\max }$ was calculated from direction discrimination errors in a two-flash apparent motion sequence and was estimated as the displacement at which there were $20 \%$ errors.

\section{Results}

Figure 2 shows $d_{\max }$ as a function of ISI for 2 observers. For Observer P.B. there was a gradual decrease in $d_{\max }$ with ISI, but for Observer I.M., there was a slight increase. Taken together, the 2 observers' data did not indicate any consistent dependence of $d_{\max }$ on ISI, in spite of the quite different subjective percept at large ISIs. This result is likely to be due to the constraints imposed by the geometry of stimulus construction rather than limitations of visual processing: The estimates of $d_{\max }$ were around half the mean interpattern separation of the micropatterns, $2.7^{\circ}$.

Figure 3 shows $d_{\min }$ as a function of ISI for 2 observers. While the absolute values of $d_{\min }$ differed somewhat between the observers, the pattern of dependence on ISI was similar. For both observers, $d_{\min }$ was approximately invariant at relatively short ISIs (up to around $100 \mathrm{msec}$ ), but when the ISI exceeded about $100 \mathrm{msec}$, there was a steady increase in $d_{\text {min. }}$. This break correlated with the observers' subjective percept of the stimulus, giving a strong sensation of movement at shorter but not at longer ISIs. Unlike $d_{\max }, d_{\min }$ was not constrained by the geometry of the stimulus.

\section{EXPERIMENT 2 \\ The Effect of Distractor Elements on Direction Discrimination}

In Experiment 2, we measured the effects of masking on direction discrimination. Method was as described in the General Method section, except that masking "distrac- 


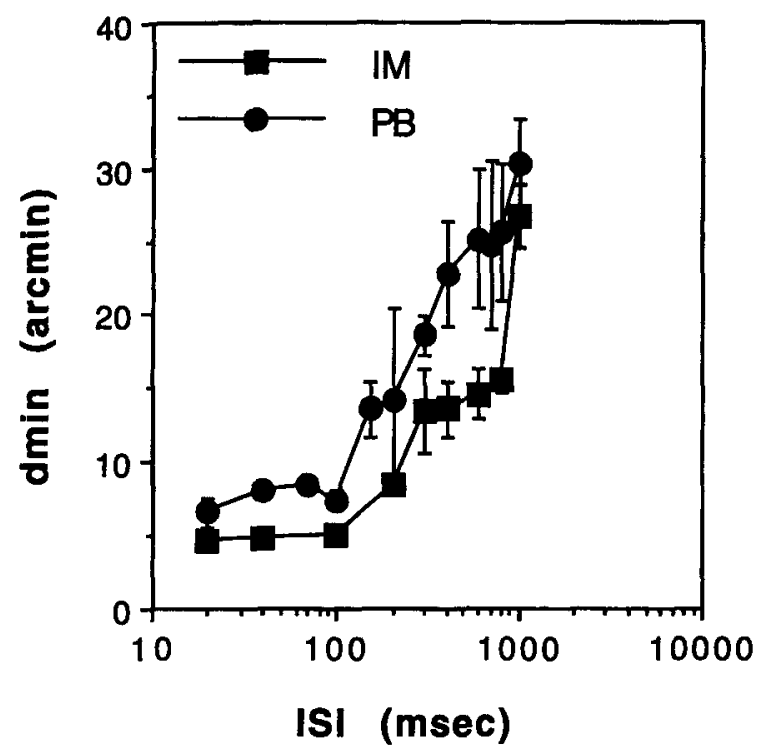

Figure 3. $d_{\min }$ as a function of interstimulus interval (ISI) for 2 observers. $d_{\min }$ was calculated as for $d_{\max }$ (see text and Figure 2) and was again estimated as the displacement at which there were $20 \%$ errors.

tor" micropatterns ( 3 per row, 12 per flash) were added to one or both flashes. An illustration of the appearance of one frame of the display containing distractors together with targets is shown in Figure 4B. The distractors were randomly positioned in the stimulus areas of the display, but were not displaced coherently with the target micropatterns. If present on both flashes, the distractors were either in the same position on the second flash (static distractors) or were plotted to a new random location on the second flash (dynamic distractors). In a separate condition, the distractors were presented on only one flash. This ensured that the distractors (particularly when static) could not be used as static reference points from which to determine the displacement of the targets. There were three targets (coherently displaced micropatterns) per row, which were displaced between flashes by one quarter of the mean interpattern separation $\left(1.3^{\circ}\right)$. This displacement resulted in fewest errors in direction discrimination across a range of ISIs when no distractors were present. The number of direction discrimination errors was recorded as a function of ISI.

\section{Results}

Direction discrimination errors are shown as a function of ISI for both observers in Figures 5 and 6. In Figure 5 , the distractors were present on both flashes, whereas in Figure 6 the distractors were present on only one flash. When there were no distractors (open triangles, broken lines), observers were able to indicate the direction of displacement for a very broad range of ISIs up to about $500 \mathrm{msec}$, after which the error rate began to rise slightly. However, in all conditions when distractors were present, the pattern of results was different. For ISIs shorter than approximately $100 \mathrm{msec}$, there were few errors, but for longer ISIs, the error rate rose steadily and direction discrimination fell rapidly to chance levels. This trend was independent of whether the distractors were present on both flashes or only one flash, showing that the distractors did not serve as reference points from which to judge the direction of motion. The results demonstrate that distractors selectively interfere with, or mask, direction discrimination at larger ISIs, but not for ISIs below about $100 \mathrm{msec}$. It is unlikely that this result is a "floor effect" at low ISIs because error rates were slightly above zero even at ISIs between 8 and $100 \mathrm{msec}$.

\section{EXPERIMENT 3 \\ The Effect of Distractor Density on Direction Discrimination}

The results of Experiments 1 and 2, showing a discontinuity in psychophysical performance at an ISI of about $100 \mathrm{msec}$, indicate that the nature of direction discrimination performance is categorically different for ISIs below and above $100 \mathrm{msec}$. In Experiment 3, we measured the effects of varying distractor density at two values of ISI, on either side of this cutoff. Errors in direction discrimination were recorded as described in the General Method section for an ISI of either 40 or $500 \mathrm{msec}$. There were three target micropatterns per row and the number of distractors was varied. An illustration of the appearance of one frame of the display containing targets together with three, six, or nine distractors per row is shown in Figure 4B-4D. Distractors were either dynamic (i.e., they were randomly repositioned between flashes) or static (i.e., they were presented in the same location on both flashes). The target micropatterns were again displaced between flashes by one quarter of the mean interpattern separation.

\section{Results}

Direction discrimination errors are presented as a function of distractor density for both observers in Figure 7. It can be seen that once again the behavior at short and long ISIs was different. At a long ISI (open symbols), direction discrimination climbed steeply to chance levels with the introduction of even a few distractors and was at nearly chance levels when the number of distractors exceeded the number of targets. The effect was independent of whether the distractors were dynamic or static. At a short ISI (closed symbols), distractors also degraded direction discrimination, but in a much more gradual manner. For dynamic distractors, direction discrimination was well above chance even when there were three or four times as many distractors as targets. When the distractors were static, for 1 observer (P.B.) there was a slight increase in errors (to about $15 \%$ for any number of distractors), but this error rate was constant for all distractor densities measured (up to $48=12$ per row). For the 2 nd observer (I.M.), the error rate rose gradually with distractor density for both static and dynamic distractors. Despite the 

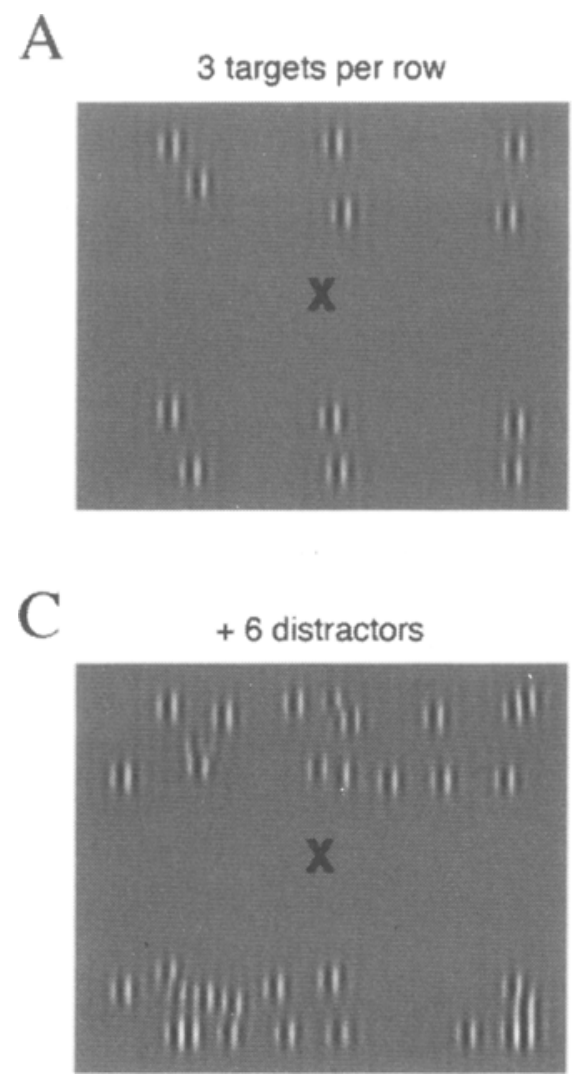
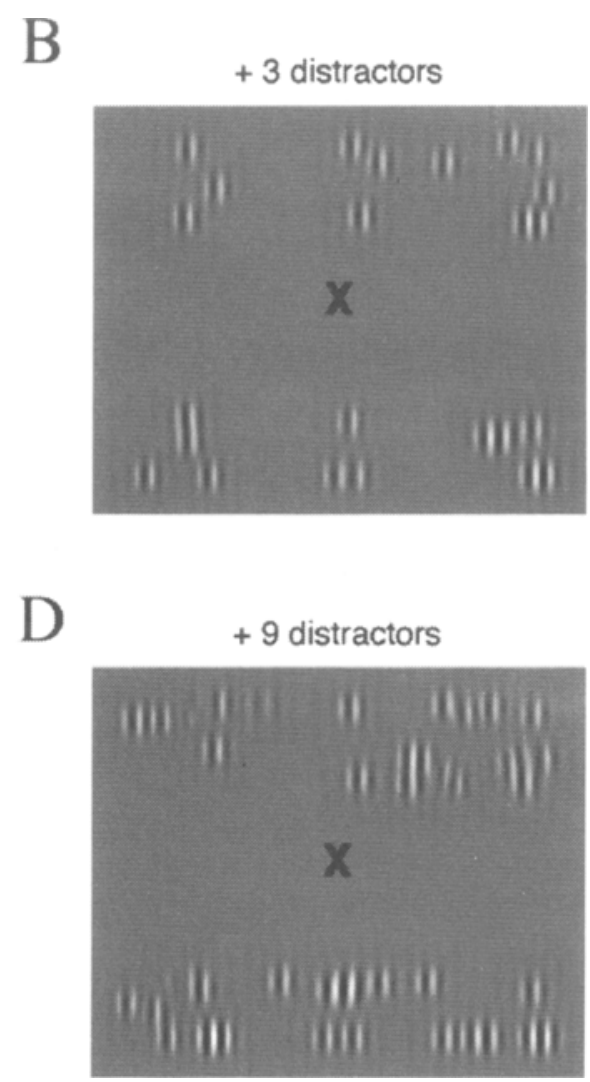

Figure 4. Spatial layout of the stimulus containing target and distractor micropatterns. Two rows of pseudorandomly placed Gabor micropatterns were placed in strips above and below a central fixation point. A: Three target micropatterns per row, no distractors. B: Three distractors per row have been added to the display, according to the same pseudorandom rules. In $C$, six distractors and in $D$, nine distractors have been added.

variability between subjects, the dependence on distractor density is manifestly different at short and long ISIs.

\section{GENERAL DISCUSSION}

The results show that direction discrimination is possible when even very long ISIs are introduced in the displacement of a two-flash apparent motion kinematogram. In Experiment 1, it was found that the upper displacement limit of motion, $d_{\max }$, was relatively unaffected by ISI. However, the lower displacement limit, $d_{\min }$, was greatly dependent on ISI: For ISIs shorter than about $100 \mathrm{msec}$, observers were sensitive to small displacements, but rapidly became less sensitive with further increases in ISI. In Experiments 2 and 3, the addition of randomly placed masking elements (distractors) was found to not affect performance when the ISI was below about $100 \mathrm{msec}$, but at longer ISIs, performance fell rapidly to chance levels. Furthermore, at a short ISI ( $40 \mathrm{msec}$ ), motion perception was relatively insensitive to distractors, but at a longer ISI $(500 \mathrm{msec})$, direction discrimination fell to chance levels with the addition of very few distrac- tors. Note that the ISI at which the transition occurred $(100 \mathrm{msec}$ ) was consistent across observers and between the two somewhat different tasks of minimum displacement detection in Experiment 1 and direction discrimination in the presence of static or dynamic distractors in Experiment 2.

Collectively these results suggest that motion perception is supported by categorically distinct processes at short and long ISIs and that the transition between them occurs when the ISI exceeds approximately $100 \mathrm{msec}$. At short ISIs, motion detection is sensitive to small spatial displacements and is relatively insensitive to distractor masking, but at long ISIs motion detection is insensitive to small spatial displacements and highly vulnerable to distractors. The lack of effect of distractors at short ISIs suggests that this mechanism is energy based at some stage because distractor micropatterns have only a limited effect on the net orientation of motion energy (Bex \& Baker, 1997). At longer ISIs, however, distractor micropatterns seriously disrupt motion perception, suggesting that the distractors present false correspondences between targets on the two flashes of apparent motion, consistent 

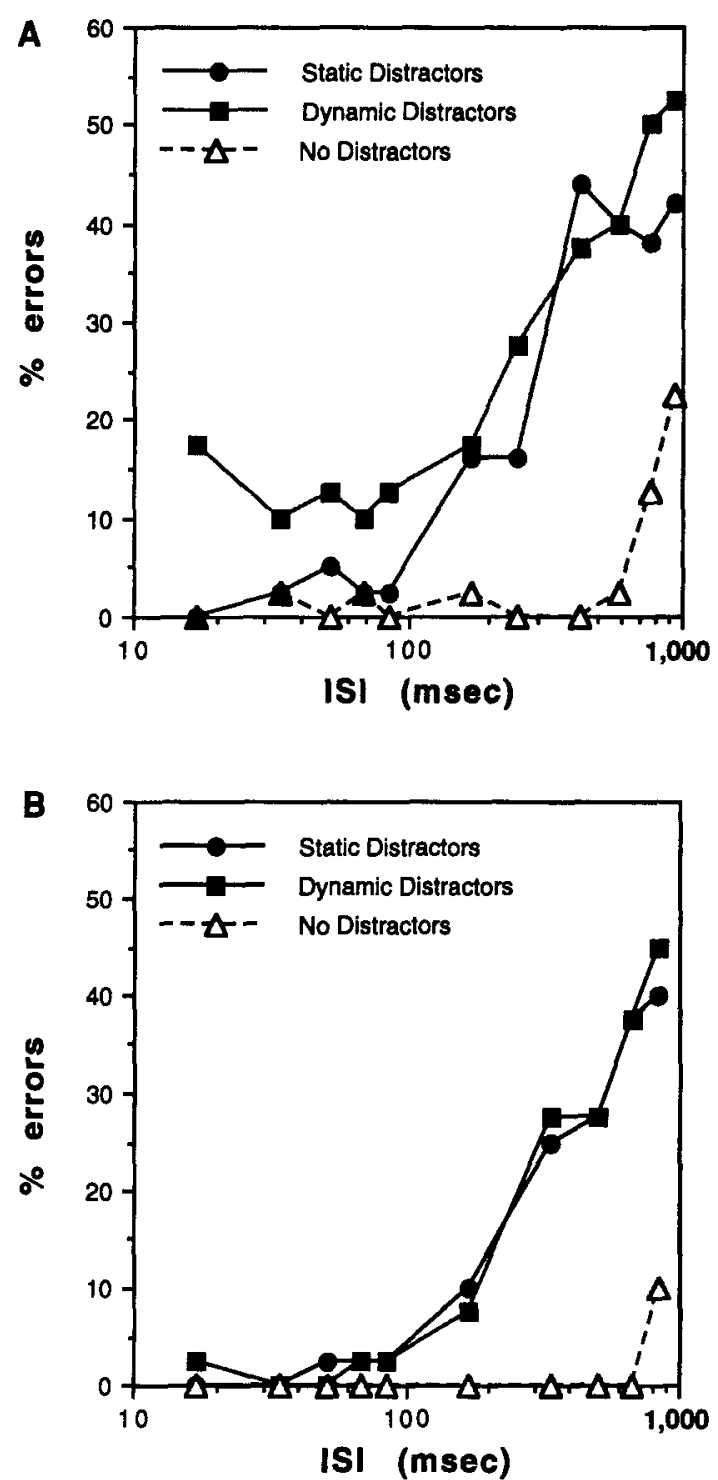

Figure 5. Direction discrimination in a two-flash apparent motion display in which there were randomly positioned distractors present on both flashes in addition to the target micropatterns. The data for the 2 observers is shown separately in panels A (P.B.) and $B$ (I.M.). There were three targets and three distractors per row (12 each per flash). Each data point is the mean of at least 40 observations.

with a high-level strategy based on, for example, nearestneighbor feature matching (Ullman, 1979) or a perceived change of remembered position (Palmer, 1986).

\section{Relation to Previous Studies}

Two studies have shown that the apparent direction of compound gratings can vary with the introduction of a long ISI (Georgeson \& Harris, 1990; Smith, 1994). Both Georgeson and Harris and Smith designed stimuli whose features moved in the opposite direction to the motion energy (at a particular spatial scale in the stimulus) with a quarter-cycle shift of the image. The patterns were either a missing fundamental grating (Georgeson \& Harris, 1990) or a beat at the difference frequency between two contrast-modulated gratings (in this case, motion energy was present only after a nonlinear rectification stage, Smith, 1994). Both studies showed that without an ISI, the perceived direction was consistent with the analysis of motion energy (after rectification for the second-order case), but with an ISI greater than about $50 \mathrm{msec}$, the perceived direction was consistent with the shortest displacement between features in the compound gratings. These results were taken as evidence that at short ISIs, motion perception is supported by energy-based mecha-
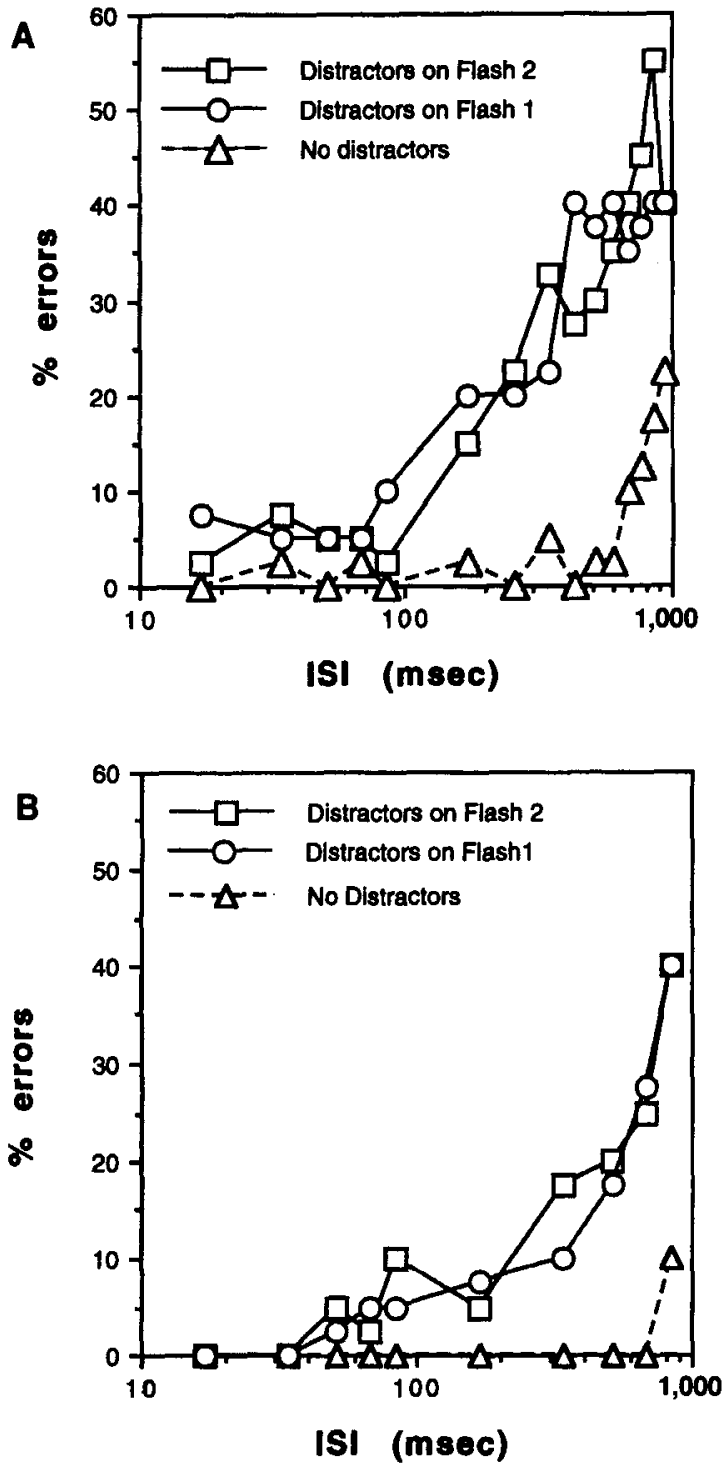

Figure 6. Direction discrimination in a two-flash apparent motion display in which there were randomly positioned distractors present on only one flash. The data for the 2 observers are shown separately in panels $A$ (P.B.) and B (I.M.). There were three targets and three distractors per row (12 each per flash). Each data point is the mean of at least $\mathbf{4 0}$ observations. 
A
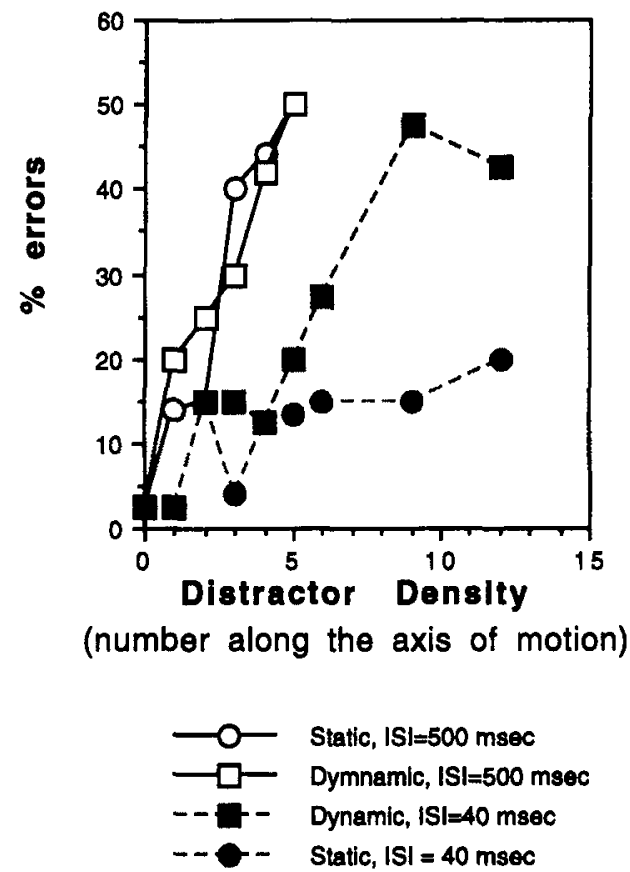

B

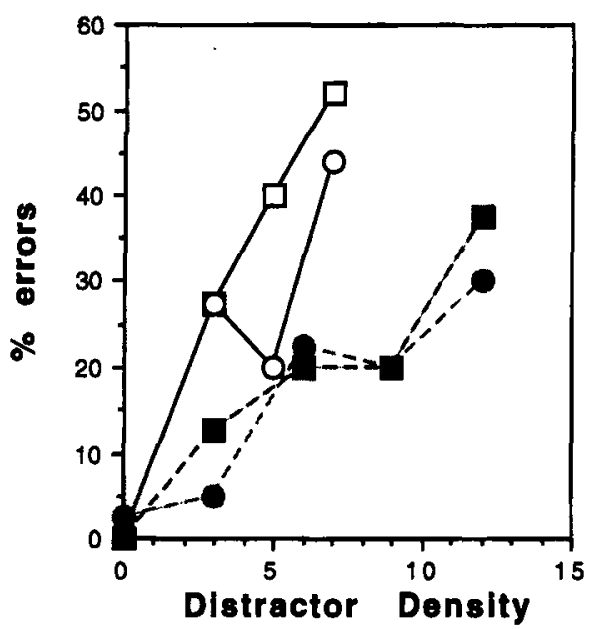

Figure 7. Direction discrimination as a function of distractor density at short or long interstimulus intervals (ISIs). The data for the 2 observers are shown separately in panels $A$ (P.B.) and $B$ (I.M.). There were three targets per row (12 per flash) on each flash; the number of distractors along the axis of motion is shown on the abscissa (the total number of distractors per flash is four times the number along the axis of motion). Each data point is the mean of at least $\mathbf{4 0}$ observations.

nisms, but at longer ISIs, motion perception is supported by feature-based mechanisms. Our results support the general conclusions of these studies (that the mechanisms supporting motion perception change as ISI increases) but in the present study, the transition occurred at a somewhat longer ISI of $100 \mathrm{msec}$.

The reversal in the apparent direction of motion with an ISI in these studies could be accounted for by lowlevel motion energy models with a biphasic temporal im- pulse response (see, e.g., Strout, Pantle, \& Mills, 1994). Such models predict the changes in apparent direction of compound gratings with an ISI without invoking separate mechanisms. However, such variants of energybased models do not predict the substantial increase in displacement limits with ISI and element density (Boulton \& Baker, 1993a, 1993b, 1994). Likewise, the addition of a rectifying nonlinearity to such models (to detect the displacement of the micropattern's envelope) would not account for the changes in $d_{\min }$ with ISI that we reported in Experiment 1, or for the greater vulnerability to distractors at longer ISIs (Experiments 2 and 3 ).

Other researchers have also argued that three mechanisms support motion perception by human observers (Lu \& Sperling, 1995b). A variety of stimuli and paradigms (including interocular presentation and attentional manipulations) were combined to reveal distinct motion processing mechanisms. One procedure was based on the idea that the presence of a static masking grating should not affect the response of motion energy detectors to a simultaneous drifting grating. Recent psychophysical evidence questions this assumption (Chaparro, Stromeyer, Kronauer, \& Cavanagh, 1996), but the present data support the overall conclusion that there exist two low-level energy-based mechanisms distinct from a third, higher level, feature-tracking mechanism. Recently, Ho (1998) studied motion perception mechanisms using a complex moving pattern (Werkhoven et al., 1993), in which the direction of second-order components is opposite to that of third-order (feature tracking) components. Ho found that simultaneously performing a second-order motion detection task selectively impaired second-order motion perception, but spared feature-tracking. Likewise, simultaneously performing an attentional task (letter recognition or another third-order motion task) selectively impaired feature tracking but spared second-order motion perception. Our results support her suggestion that two nonlinear mechanisms support motion perception.

Fahle and Harris (1992) have also studied visual sensitivity over long ISIs in a Vernier acuity task. Observers were presented with two Vernier patterns in two intervals separated by up to $8 \mathrm{sec}$ and were required to indicate the interval containing the pattern with greatest offset. This task eliminated the problem of cumulative error-for example, from eye movements during the ISI, which can easily be confounded with the decay of visual memory. It was found that thresholds rose linearly with the spatial offset of the standard pattern and also rose with ISI duration. The results were taken as direct evidence of fading of visual memory. It is possible that such fading of visual memory might account for the present results; for example, the increase in $d_{\min }$ with ISI could be the consequence of some gradual fading of visual memory of the spatial locations of elements in the first interval. But it is difficult to see why $d_{\max }$ should not be similarly affected. Likewise, this hypothesis does not predict the change of slope of direction discrimination errors as a function of distractor density at short and long ISIs. 
Unfortunately, the present direction discrimination task does not eliminate the contribution of cumulative errors, such as eye movements, to the reduction of motion perception in our tasks. The motion analogue of Fahle and Harris's (1992) task might be to discriminate the interval containing the larger jump size over a range of ISIs. Although this would eliminate the role of uncertainty from eye movements during the ISI, other variables would be introduced into the task, such as speed changes.

\section{Conclusions}

Using random Gabor kinematograms, variations in $d_{\max }$ with element density have previously been used to reveal the existence of two motion mechanisms: a quasilinear motion energy mechanism and a separate, nonlinear mechanism (Boulton \& Baker 1993a, 1993b, 1994). The present results, using the same stimuli, suggest that there are in fact two motion detection mechanisms under the "nonlinear viewing conditions" (large SOA, low density). One mechanism, operating at ISIs below $100 \mathrm{msec}$, is likely to be low level, and could be modeled as a nonlinear (envelope-extracting) stage, followed by motion energy detection at a large spatial scale (see, e.g., Bex \& Baker, 1997). The mechanism operating at larger ISIs is likely to be higher level and involve a perceived change of position of salient features, which is very easily disrupted by noncoherently moving, distracting features. Together with earlier studies showing separate quasilinear and nonlinear mechanisms (Boulton \& Baker 1993a, 1993b, 1994), the results support proposals that three mechanisms support motion perception by human observers (Ho, 1998; Lu \& Sperling, 1995b).

\section{REFERENCES}

Adelson, E. H., \& BERGEN, J. R. (1985). Spatio-temporal energy models for the perception of motion. Journal of the Optical Society of America, 2, 284-299.

AlBright, T. D. (1992). Form-cue invariant motion processing in primate visual cortex. Science, 255, 1141-1143.

BAKER, C. L., JR., \& Hess, R. F. (1998). Two mechanisms underlie processing of stochastic motion stimuli. Vision Research, 38, 1211 1222.

BEX, P. J., \& BAKER, C. L. (1997). The effects of distractor elements on direction discrimination in random Gabor kinematograms. Vision Research, 37, 1761-1767.

Bischof, W. F., \& Di Lollo, V. (1991). On the half-cycle displacement limit of sampled directional motion. Vision Research, 31, 649-660.

Boulton, J. C., \& BAKER, C. L. (1993a). Dependence on stimulus onset asynchrony in apparent motion: Evidence for two mechanisms. $\mathrm{Vi}$ sion Research, 33, 2013-2019.

Boulton, J. C., \& BAKER, C. L., JR. (1993b). Different parameters control motion perception above and below a critical density. Vision Research, 33, 1803-1811.

Boulton, J. C., \& BAKER, C. L. (1994). Psychophysical evidence for both a "quasi-linear" and a "non-linear" mechanism for the detection of motion. In T. B. Lawton (Ed.), Computational vision based on neurobiology (Proceedings of SPIE, Vol. 2054, pp. 124-133). Bellingham, WA: SPIE.
Chaparro, A., Stromeyer, C. F., III, Kronauer, R. E., \& CavaNAGH, P. (1996). Effect of static gratings on motion detection thresholds. Optical Society Annual Meeting Abstracts, 13, 106.

Chubb, C., \& Sperling, G. (1988). Drift-balanced random stimuli: A general basis for studying non-Fourier motion perception. Journal of the Optical Society of America, 5, 1986-2006.

FAHLE, M., \& HaRRIS, J. P. (1992). Visual memory for Vernier offsets. Vision Research, 32, 1033-1042.

Georgeson, M. A., \& HaRris, M. G. (1990). The temporal range of motion sensing and motion perception. Vision Research, 30, 615-619.

Ho, C. E. (1998). Letter recognition reveals pathways of second-order and third-order motion. Proceedings of the National Academy of Sciences, 95, 400-404.

LeDGeway, T., \& SMith, A. T. (1995). The duration of the motion aftereffect following adaptation to first- and second-order motion. Perception, 23, 1211-1220.

LU, Z, \& SPERLING, G. (1995a). Attention generated apparent motion. Nature, 377, 237-239.

LU, Z, \& SPERLING, G. (1995b). The functional architecture of human visual motion perception. Vision Research, 35, 2697-2722.

Mather, G., \& WEST, S. (1993). Evidence for second-order motion detectors. Vision Research, 33, 1109-1112.

NAKAYAMA, K. (1985). Biological image motion processing: A review. Vision Research, 25, 625-660.

NisHida, S. (1993). Spatiotemporal properties of motion perception for random-check contrast modulations. Vision Research, 33, 633-645.

Nishida, S., Ledgeway, T., \& EDwards, M. (1997). Dual multiple-scale processing for motion in the human visual system. Vision Research, 37, 2685-2698.

Nishida, S., \& SATo, T. (1995). Motion aftereffect with flickering test patterns reveals higher stages of motion processing. Vision Research, 35, 477-490.

Palmer, J. (1986). Mechanisms of displacement discrimination with a visual reference. Vision Research, 26, 1939-1947.

PELLI, D. G. (1997). The VideoToolbox software for visual psychophysics: Transforming numbers into movies. Spatial Vision, 10, 437-442.

Pelli, D. G., \& ZhaNG, L. (1991). Accurate control of contrast on microcomputer displays. Vision Research, 31, 1337-1350.

SMITH, A. T. (1994). Correspondence-based and energy-based detection of second-order motion in human vision. Journal of the Optical Society of America $A, 11,1940-1948$.

Strout, J. J., Pantle, A., \& Mills, S. L. (1994). An energy model of interframe interval effects in single-step apparent motion. Vision Research, 34, 3223-3240.

Ullman, S. (1979). The interpretation of visual motion. Cambridge, MA: MIT Press.

van Santen, J. P., \& Sperling, G. (1985). Elaborated Reichardt detectors. Journal of the Optical Society of America A, 2, 300-321.

Watson, A. B., \& Ahumada, A. J. (1985). Model of human visualmotion sensing. Journal of the Optical Society of America A, 2, 322-342.

WEIBULl, W. (1951). A statistical distribution function of wide applicability. Journal of Applied Mechanics, 18, 292-297.

Werkhoven, P., Sperling, G., \& ChubB, C. (1993). The dimensionality of texture defined motion: A single channel theory. Vision Research, 33, 463-485.

Wilson, H. R., Ferrera, V. P., \& Yo, C. (1992). A psychophysically motivated model for two-dimensional motion perception. Visual Neuroscience, 9, 79-97.

ZHOU, Y. X., \& BAKER, C. L., JR. (1993). A processing stream in mammalian visual cortex neurons for non-Fourier responses. Science, 261, 98-101.

Zlhl, J., Von Cramon, D., \& MaI, N. (1983). Selective disturbance of movement vision after bilateral brain damage. Brain, 106, 313-340.

(Manuscript received January 15, 1998; revision accepted for publication June $18,1998$. 